Research Article

\title{
Comparation of Anti-Inflammatory and Antioxidantactivities of Curcumin, Tetrahydrocurcuminand Octahydrocurcuminin LPS-Stimulated RAW264.7 Macrophages
}

\author{
Qing-Feng Xie $\mathbb{D}^{1,},{ }^{1,2}$ Juan-Juan Cheng $\mathbb{D},{ }^{3}$ Jin-Fen Chen, ${ }^{3}$ Yu-Chao Feng, ${ }^{1,2}$ Guo-Shu Lin, ${ }^{3}$ \\ and Yang $\mathrm{Xu}^{1,2}$ \\ ${ }^{1}$ The Second Clinical College of Guangzhou University of Chinese Medicine, Guangzhou 510006, China \\ ${ }^{2}$ Guangdong Provincial Hospital of Chinese Medicine, Guangzhou 510006, China \\ ${ }^{3}$ Guangdong Provincial Key Laboratory of New Drug Development and Research of Chinese Medicine, \\ Mathematical Engineering Academy of Chinese Medicine, Guangzhou University of Chinese Medicine, \\ Guangzhou 510000, China \\ Correspondence should be addressed to Qing-Feng Xie; xieqingfeng1@gzucm.edu.cn
}

Received 10 September 2020; Revised 13 November 2020; Accepted 1 December 2020; Published 22 December 2020

Academic Editor: Li Zhang

Copyright (C) 2020 Qing-Feng Xie et al. This is an open access article distributed under the Creative Commons Attribution License, which permits unrestricted use, distribution, and reproduction in any medium, provided the original work is properly cited.

\begin{abstract}
Curcumin (CUR) possesses pronounced anti-inflammatory and antioxidant activities. Generally, the clinical application of CUR is restricted due to its apparent unstability and poor absorption, and the biological activities of CUR may be closely associated with its metabolites. Tetrahydrocurcumin (THC) and octahydrocurcumin (OHC) are two major hydrogenated metabolites of CUR with appreciable biological potentials. Here, we comparatively explored the anti-inflammatory and antioxidant activities of CUR, THC, and OHC in lipopolysaccharide- (LPS-) induced RAW264.7 macrophages. The results revealed that CUR, THC, and OHC dose-dependently inhibited the generation of NO and MCP-1 as well as the gene expression of MCP-1 and iNOS. Additionally, CUR, THC, and OHC significantly inhibited NF- $\kappa$ B activation and p38MAPK and ERK phosphorylation, while substantially upregulated the Nrf2 target gene expression (HO-1, NQO-1, GCLC, and GCLM). Nevertheless, zinc protoporphyrin (ZnPP), a typical HO-1 inhibitor, significantly reversed the alleviative effect of CUR, THC, and OHC on LPS-stimulated ROS generation. These results demonstrated that CUR, THC, and OHC exerted beneficial effect on LPS-stimulated inflammatory and oxidative responses, at least partially, through inhibiting the NF- $\kappa$ B and MAPKs pathways and activating Nrf2-regulated antioxidant gene expression. Particularly, THC and OHC might exert superior antioxidant and anti-inflammatory activities to CUR in LPSstimulated RAW264.7 cells, which can be further explored to be a promising novel effective agent for inflammatory treatment.
\end{abstract}

\section{Introduction}

Turmeric derives from the root of Curcuma longa (Zingiberaceae), which is widely used for the therapy of various inflammatory diseases [1]. Curcumin (CUR, $\mathrm{C}_{21} \mathrm{H}_{20} \mathrm{O}_{6}$, Figure 1(a)), a vital active component derived from the rhizome of turmeric, is a common food pigment extensively used in various food. Accumulating evidence has indicated that CUR exerts an array of health-beneficial effects in vitro and in vivo [2-5]. However, CUR presents a poor systemic bioavailability due to its apparent low absorption and rapid metabolism in vivo $[6,7]$. Therefore, its therapeutic benefits may be attributed to the role of metabolites [8]. There are many important metabolites of CUR in vivo, of which tetrahydrocurcumin (THC, $\mathrm{C}_{21} \mathrm{H}_{24} \mathrm{O}_{6}$, Figure 1(b)) and octahydrocurcumin (OHC, $\mathrm{C}_{21} \mathrm{H}_{28} \mathrm{O}_{6}$, Figure $1(\mathrm{c})$ ) are its primary and final hydrogenated metabolites, respectively. Structurally, although CUR and THC possess identical phenolic groups, THC lacks $\alpha, \beta$ dienes [9]. Besides, compared to CUR, OHC also lacks $\alpha, \beta$ dienes, while it has more phenolic groups [10]. Furthermore, they have been reported to possess stronger pharmacological and biological effects against various diseases such as anti-inflammatory [10,11], antidiabetic [12], and antioxidant properties [13-16]. Nevertheless, current 
research studies on the comparative anti-inflammatory and antioxidant effects of CUR, THC, and OHC are very limited and insufficient. Therefore, it is of great importance to examine and compare the anti-inflammatory and antioxidant activities of CUR with its two metabolites of THC and OHC in LPS-stimulated RAW264.7 cells.

Inflammation, an important natural immune response, occurs when our body suffers from pathogen attacks or other harmful stimuli $[17,18]$. Therefore, it is indispensable to have a moderate inflammatory response in host innate defense system to fight against adverse infection. However, uncontrolled or continuous response would cause the abnormal expression of typical proinflammatory cytokines, which increase the risk of many complicated diseases such as asthma [19], pulmonary diseases [20], and rheumatoid arthritis [21]. In order to maintain homeostasis, inflammatory response is regulated by multiple immune cells including macrophages and lymphocytes. As the first line of the host defense, macrophages are of significance to mediate inflammation. The stimulation of lipopolysaccharide (LPS), a major endotoxin component of Gram-negative bacteria in the outer membrane, contributes considerably to increasing the productions of multiple proinflammatory cytokines $[22,23]$.

It has been widely known that nuclear factor $-\kappa \mathrm{B}(\mathrm{NF}-\kappa \mathrm{B})$ is a typical pathway, which participates in inflammatory process via releasing excessive monocyte chemotactic protein-1 (MCP-1), nitric oxide (NO), inducible nitric oxide synthase (iNOS), and other inflammatory cytokines [24, 25]. Mitogen-activated protein kinases (MAPKs) are critical to cell growth, differentiation, as well as proliferation, which are also responsible for the gene expression of inflammatory mediators [25]. Additionally, oxidative stress occurred when the steady-state of cellular redox is disrupted, which is always observed during the inflammatory response [26, 27]. Increasing evidence has suggested that a number of detoxification and antioxidant enzymes regulated by Nrf2 have imperative effect on protecting and fighting against oxidative stress, including heme oxygenase-1 (HO-1), glutamatecysteine ligase catalytic (GCLC), modifier subunits (GCLM), and $\mathrm{NAD}(\mathrm{P}) \mathrm{H}$ : quinone oxidoreductase-1 (NQO1) [28, 29]. Interestingly, many investigations have shown that the three signaling pathways are closely related to each other $[30,31]$. Therefore, a complete analysis of the interrelationships between different pathways involved in LPS-induced RAW264.7 macrophages is essential. Taken together, dual antioxidant and inhibition of NF- $\kappa$ B or MAPKs pathway could be a feasible strategy to prevent and treat inflammatory diseases. Therefore, the current investigation aimed to comparatively explore the anti-inflammatory and antioxidant activities of CUR, THC, and $\mathrm{OHC}$ and the potential role of $\mathrm{NF}-\kappa \mathrm{B} / \mathrm{MAPK}$ signal pathways in LPS-induced RAW264.7 macrophages.

\section{Materials and Methods}

2.1. Materials and Reagents. OHC (>98\% pure) was synthesized as we previously described [32]. CUR, THC, and LPS were all purchased from Sigma-Aldrich (St. Louis, Mo,
USA). The test articles were dissolved in dimethyl sulfoxide (DMSO), and the solvent concentration was ensured to be $<0.1 \%$ in all experiments [33]. Greiss reagent and enzymelinked immunosorbent assay (ELISA) kits for reactive oxygen species (ROS) were purchased from Beijing Cheng Lin Biotechnology Technology Co., Ltd. (Beijing, China). The following primary antibodies for Western blot: NF- $\kappa \mathrm{B}$ (\#8242), MAPK-p-p38 (\#4511), MAPK-p-ERK (\#8690), $\beta$-tubulin, and secondary antibodies were purchased from Cell Signaling Technology MA, USA. The primer sequences for MCP-1, iNOS, HO-1, NQO-1, GCLC, and GCLM were from Sangon Biotech Co., Ltd. (Shanghai, China). Dulbecco's modified Eagle's medium (DMEM) and fetal bovine serum (FBS) were purchased from Gibco (Shanghai, China) and GE Healthcare (Yauranga, New Zealand), respectively.

2.2. Cell Culture and Cell Viability. RAW264.7 macrophage cells were obtained from the American Type Cell Collection (ATCC; Manassas, VA, USA). Cells were cultured in DMEM supplemented with $10 \% \mathrm{FBS}, 100 \mathrm{U} / \mathrm{mL}$ penicillin, and $100 \mathrm{~g} / \mathrm{mL}$ streptomycin at $37^{\circ} \mathrm{C}$ under an atmosphere of $5 \%$ $\mathrm{CO}_{2}$. CellTiter 96 AQueous One Solution Cell Proliferation Assay (Promega Corporation, USA) was used to measure the effects of CUR, THC, and OHC on cell viability, as described previously [34]. In brief, RAW264.7 macrophage cells $\left(1 \times 10^{6}\right.$ cells $\left./ \mathrm{mL}\right)$ were seeded in a 96-well plate. After cultivation overnight, fresh medium with various concentrations of test articles was added to each well, followed by incubation for $24 \mathrm{~h}$ or $48 \mathrm{~h}$. Thereafter, the medium was removed, and $20 \mu \mathrm{L}$ solution was added to each plate. The cells were incubated for additional $4 \mathrm{~h}$, and the absorbance value was spectroscopically measured at $490 \mathrm{~nm}$. The cell viability compared to control was calculated as follows: (A drug group-A blank group)/(A control group-A blank group) $\times 100 \%$.

2.3. Nitric Oxide (NO) Assay. RAW264.7 cells $\left(1 \times 10^{6}\right.$ cells/ $\mathrm{mL}$ ) were seeded on to a 96-well plate for $24 \mathrm{~h}$. The cells were then incubated with or without CUR, THC, and OHC (2, 4, $8 \mu \mathrm{M})$ for $2 \mathrm{~h}$. The dosages were selected based on our prior trial. Subsequently, cells were added with LPS $(100 \mathrm{ng} / \mathrm{mL})$ for $24 \mathrm{~h}$. Griess reagent kit was used to measure NO generation. And the assay was performed according to the manufacturer's instruction.

2.4. Measurement of Monocyte Chemotactic Protein-1 (MCP1) Levels. RAW264.7 cells $\left(1 \times 10^{6}\right.$ cells $\left./ \mathrm{mL}\right)$ were incubated in a 96-well plate for $24 \mathrm{~h}$. Then, cells were preincubated with or without CUR, THC, and $\mathrm{OHC}(2,4$, and $8 \mu \mathrm{M})$ for $2 \mathrm{~h}$, which were subjected to LPS $(100 \mathrm{ng} / \mathrm{mL})$ stimulation for $24 \mathrm{~h}$. MCP-1 production in cell-free supernatants was detected using ELISA kits in accordance with the manufacturer's recommendations.

2.5. Determination of Intracellular Reactive Oxygen Species (ROS). DCFH-DA probe was used to detect ROS generation according to the manufacturer's instruction. In brief, 
<smiles>COc1cc(/C=C/C(=O)CC(=O)/C=C/c2ccc(O)c(O)c2)ccc1O</smiles>

(a)<smiles>COc1cc(CCC(=O)CC(=O)CCc2ccc(O)c(O)c2)ccc1O</smiles>

(b)<smiles>COc1cc(CCC(O)CC(O)CCc2ccc(O)c(O)c2)ccc1O</smiles>

(c)

Figure 1: The chemical structures of curcumin (a), tetrahydrocurcumin (b), and octahydrocurcumin (c).

RAW264.7 cells $\left(1 \times 10^{6}\right.$ cells $\left./ \mathrm{mL}\right)$ were seeded in a 12 -well plate and incubated overnight. Then, the cells were pretreated with or without CUR, THC and OHC $(2,4,8 \mu \mathrm{M})$ for $2 \mathrm{~h}$ before challenged by LPS $(100 \mathrm{ng} / \mathrm{mL})$. After $24 \mathrm{~h}$, the cells were added with $10 \mu \mathrm{M}$ DCFH-DA for $30 \mathrm{~min}$ in the dark, which was then collected to accurately evaluate ROS production using a fluorescence microscope (Agilent NovoCyte Quanteon, USA) with a multiplate reader at the wavelength of $485 \mathrm{~nm}$ and $525 \mathrm{~nm}$ (excitation and emission), respectively.

2.6. Quantitative Real-Time PCR. Total RNAs were extracted from RAW264.7 cells with TRIzol reagent, and its purity was evacuated according to the ratio of $\mathrm{OD}_{260 / 280}$, which should be from 1.8 to 2.0. And then, total RNAs were reversetranscribed into cDNA by the Primer Script RT reagent kit. Afterwards, real-time PCR amplification was performed with an initial predegeneration step at $95^{\circ} \mathrm{C}$ for $3 \mathrm{~min}$, followed by 39 cycles at $95^{\circ} \mathrm{C}$ for $10 \mathrm{sec}, 55^{\circ} \mathrm{C}$ for $10 \mathrm{sec}$, and $72^{\circ} \mathrm{C}$ for $30 \mathrm{sec}$, and a final single cycle at $95^{\circ} \mathrm{C}$ for $10 \mathrm{sec}$. The target gene expression levels relative to $\beta$-actin were determined using $2^{-\Delta \Delta \mathrm{Ct}}$ method. The sequences of the primers used are listed in Table 1.

2.7. Western Blot Analysis. The whole-cell lysates were prepared in RIPA (Radio-Immunoprecipitation Assay) buffer containing a cocktail of protease inhibitors for $40 \mathrm{~min}$ at $4^{\circ} \mathrm{C}$. They were further centrifuged at $12,000 \mathrm{rpm}$ for $10 \mathrm{~min}$ at room temperature. Besides, the protein content was determined by BCA protein assay kit. The equivalent proteins were then separated on $10 \%$ sodium dodecyl sulfate-polyacrylamide gel electrophoresis (SDS-PAGE) and transferred to a polyvinylidene fluoride (PVDF) membrane. Afterwards, 5\% nonfat skim milk was used to block the membrane for $1 \mathrm{~h}$, and the membrane was then covered with specific primary antibodies at $4^{\circ} \mathrm{C}$ overnight. Subsequently, PVDF membrane was incubated with secondary antibodies for $1 \mathrm{~h}$. Finally, the antibody specific bands were detected by the enhanced chemiluminescence (ECL) (Bio-Rad, ChemiDoc XRS+, USA) for 1 to $2 \mathrm{~min}$. And ImageJ (National
Table 1: Primer sequences.

\begin{tabular}{lc}
\hline Gene & Sequence $\left(5^{\prime}\right.$ to $\left.3^{\prime}\right)$ \\
\hline MCP-1 & Forward AAGTTGACCCGTAAATCTGA \\
& Reverse TGAAAGGGATACCATAACA \\
iNOS & Forward TTGCACGTGTTAAGGATGCC \\
& Reverse GTCAACGCTTGGGAGAGTGT \\
NQO1 & Forward GCGAGAAGAGCCCTGATTGT \\
& Reverse GGCGTCCTTCCTTATGTGCT \\
HO-1 & Forward AAGCCGAGAATGCTGAGTTCA \\
& Reverse GCCGTGTAGATATGGTACAAGGA \\
GCLC & Forward GGGGTGACGAGGTGGAGTA \\
& Reverse GTTGGGGTTTGTCCTCTCCC \\
GCLM & Forward AGGAGCTTCGGGACTGTATCC \\
& Reverse GGGACATGGTGCATTCCAAAA \\
$\beta$-Actin & Forward AGCCATGTACGTAGCCATCC \\
& Reverse CTCTCAGCTGTGGTGGTGAA \\
\hline
\end{tabular}

Institutes of Health, United States) was used to capture images for quantitative assessment.

2.8. Statistical Analyses. Results were presented as mean\pm standard deviation (SD). The experiments were repeated three times. The statistical of the data was carried out by oneway ANOVA followed by post hoc Dunnett's test using SPSS software (version 20.0, Chicago, IL, USA). $P<0.05$ was regarded to indicate a statistically significant difference.

\section{Results}

3.1. Assessment of Cell Toxicity of CUR, THC, and OHC in RAW264.7 Cells. As shown in Figures 2(a)-2(c), up to a concentration of $32 \mu \mathrm{M}$, CUR did not show cytotoxic effect after incubation for $24 \mathrm{~h}$ or $48 \mathrm{~h}(P>0.05)$. However, the viability of RAW264.7 cells was significantly reduced by CUR at $64 \mu \mathrm{M}$, while THC and OHC at concentrations ranging from 2 to $64 \mu \mathrm{M}$ exhibited no cytotoxic effects on the viability of RAW264.7 cells. Therefore, 2,4 , and $8 \mu \mathrm{M}$ were used in the subsequent experiments.

3.2. Evaluation of NO and MCP-1 Generation as well as $i N O S$ and MCP-1 mRNA Expression in RAW264.7 Cells. As shown 


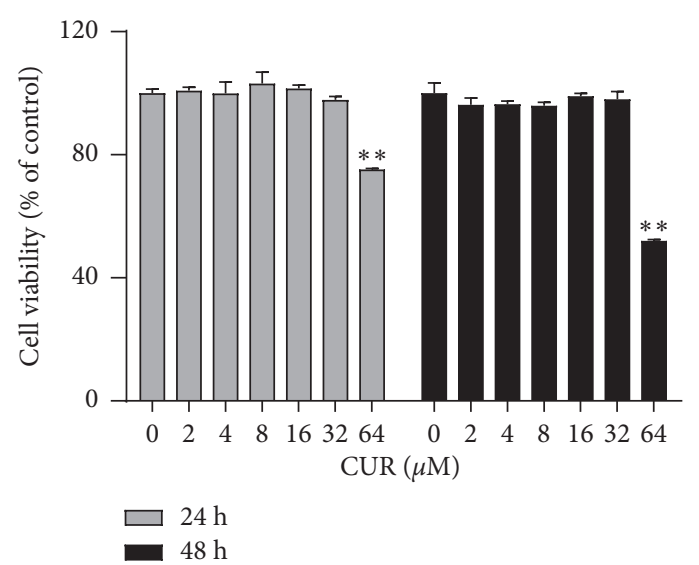

(a)

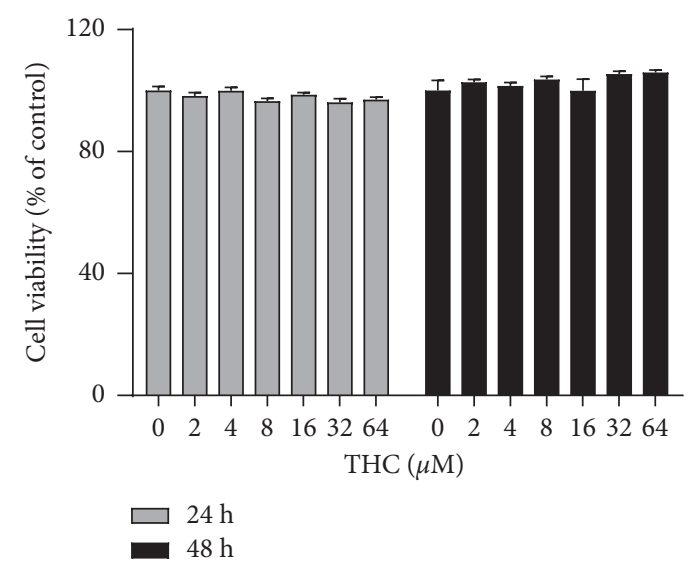

(b)

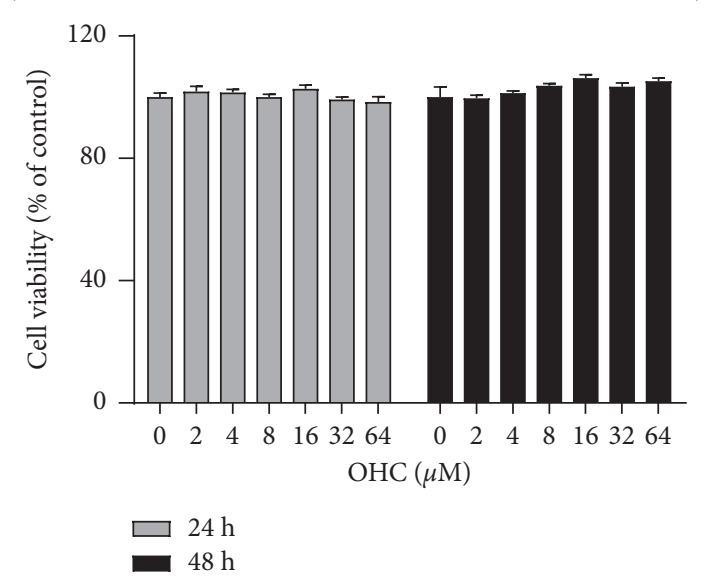

(c)

Figure 2: Effects of CUR (a), THC (b), and OHC (c) on cell viability of RAW264.7 macrophages. Data are shown as mean \pm SD $(n=3)$; ${ }^{* *} P<0.01$ vs. control group.

in Figures 3(a)-3(b), the result indicated that LPS (100 ng/ $\mathrm{mL}$ ) treatment notably increased $\mathrm{NO}$ and MCP-1 productions (all $P<0.01$ ) as compared to the control group. Nevertheless, the productions of $\mathrm{NO}$ and MCP-1 were remarkably and dose-dependently inhibited (all $P<0.01$ ) by CUR, THC, and OHC. In addition, THC and OHC were found to exhibit more pronounced (all $P<0.01$ ) inhibitory effect as compared to CUR. As shown in Figures 3(c)-3(d), compared to the control group, MCP-1 and iNOS gene expression was significantly upregulated (all $P<0.01$ ) after LPS treatment. In contrast, this pattern could be visibly suppressed (all $P<0.01$ ) by pretreating with CUR, THC, and OHC in a concentration-dependent manner. Notably, THC and $\mathrm{OHC}$ treatment also exhibited more potent effect (all $P<0.01)$ than CUR in suppressing the gene expression of MCP-1 and iNOS.

3.3. Evaluation of GCLC, GCLM, HO-1, and NQO-1 mRNA Expression in RAW264.7 Cells. As shown in Figures 4(a)4(d), the mRNA expression of HO-1, NQO-1, GCLC, and GCLM was dramatically inhibited (all $P<0.01$ ) in the LPS group, as compared to the control group. Nevertheless, pretreatment with CUR, THC, and OHC all remarkably and dose-dependently enhanced (all $P<0.01$ ) the gene expression of HO-1, NQO-1, GCLC, and GCLM, respectively. Noteworthily, THC and $\mathrm{OHC}$ showed more noticeable activities $(P<0.05$ and $P<0.01)$ than CUR in promoting the antioxidant gene expression (HO-1, NQO-1, GCLC, and GCLM).

3.4. ZnPP Blocks the Inhibitory Effect of CUR, THC, and OHC on LPS-Induced ROS Production. As shown in Figures 5(a)5(c), CUR, THC, and OHC were observed to increase the protein levels of HO-1 (all $P<0.01$ ) and dose-dependently inhibit the generation of $\operatorname{ROS}(P<0.05)$ in cells treated with LPS relative to the LPS model group. Additionally, to further confirm whether the inhibitory effect of CUR, THC, and $\mathrm{OHC}$ against intracellular ROS generation depended on HO-1, the cells were added with HO-1 inhibitor ZnPP during the treatment. Our result indicated that HO-1 protein level was found to be decreased by $\mathrm{ZnPP}$ to a large extent when compared with that of other treatment groups. Furthermore, the inhibitory effect of CUR, THC, and OHC on suppressing ROS generation was greatly reversed in the presence of $\mathrm{ZnPP}$. 


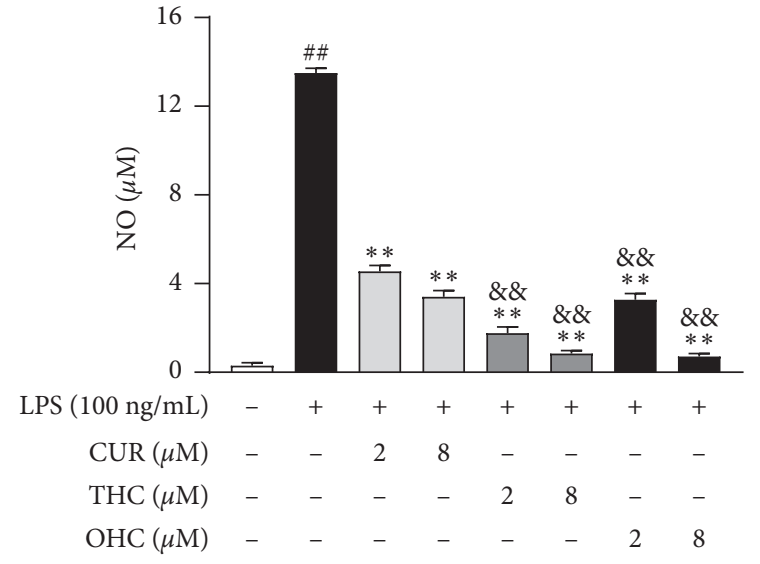

(a)

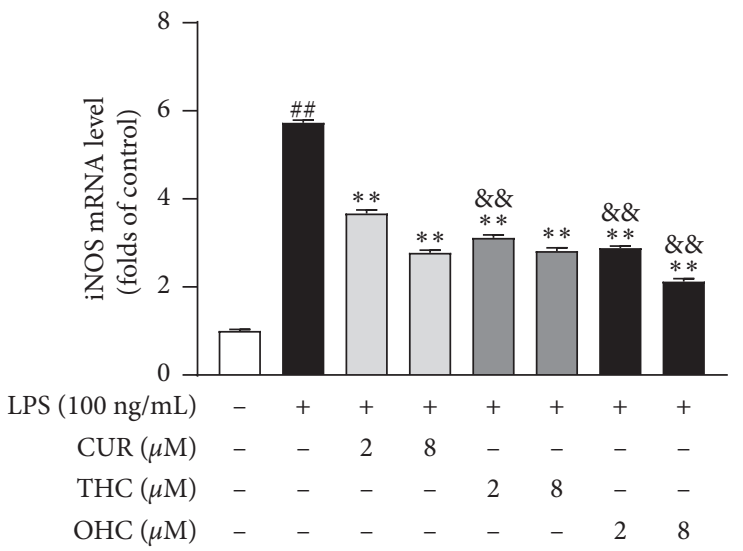

(c)

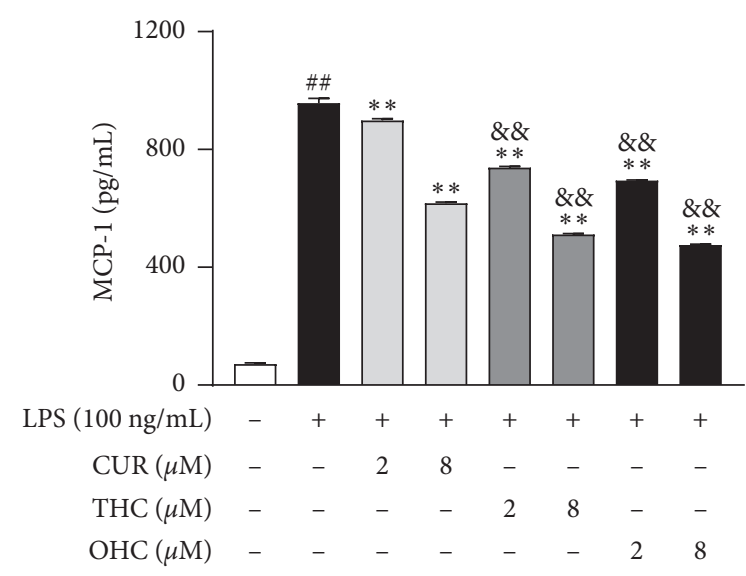

(b)

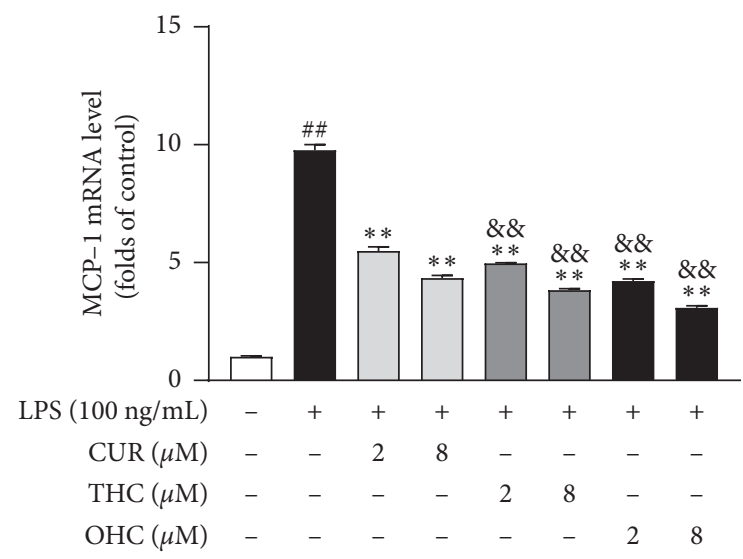

(d)

Figure 3: Effects of CUR, THC, and OHC on the productions of NO (a) and MCP-1 (b) and the mRNA expression of iNOS (c) and MCP-1 (d) in LPS-activated RAW264.7 cells. Data are shown as mean \pm SD $(n=3)$; ${ }^{\# \#} P<0.01$ vs. control group, ${ }^{* *} P<0.01$ vs. LPS group, and ${ }^{\& \&} P<0.01$ vs. CUR of the same dose.

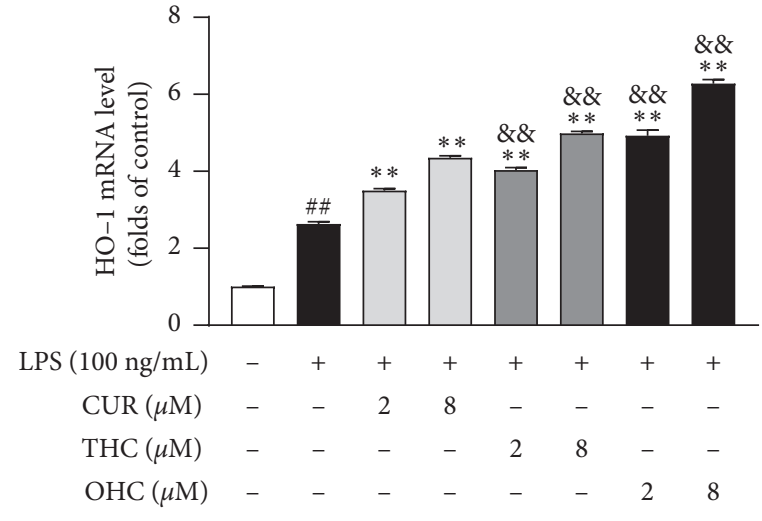

(a)

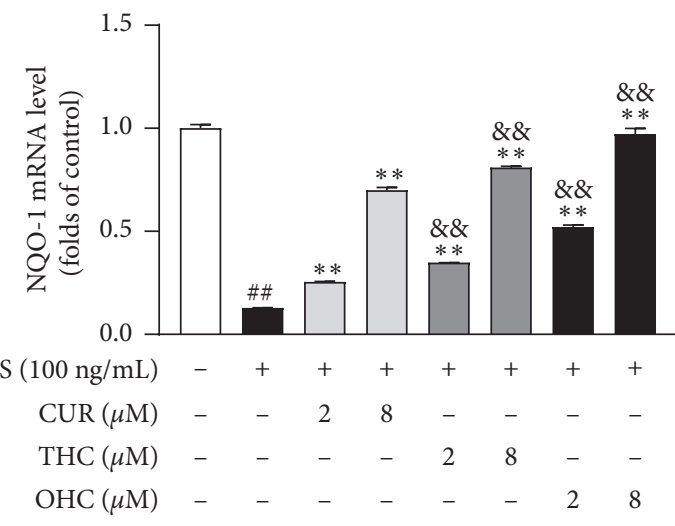

(b)

Figure 4: Continued. 


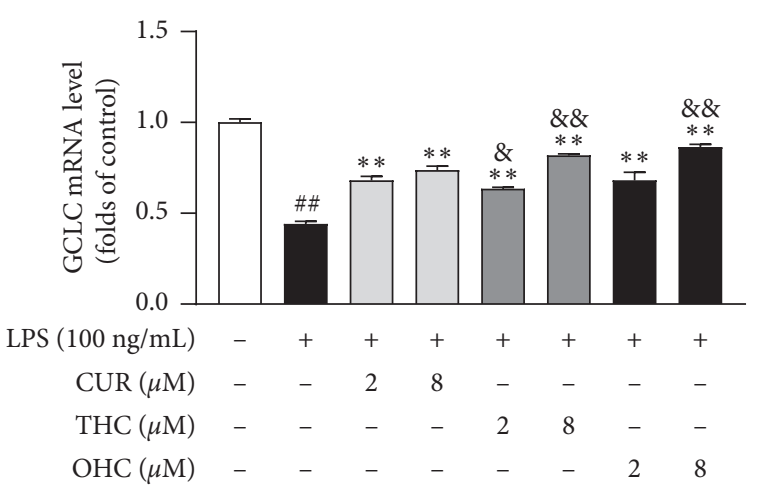

(c)

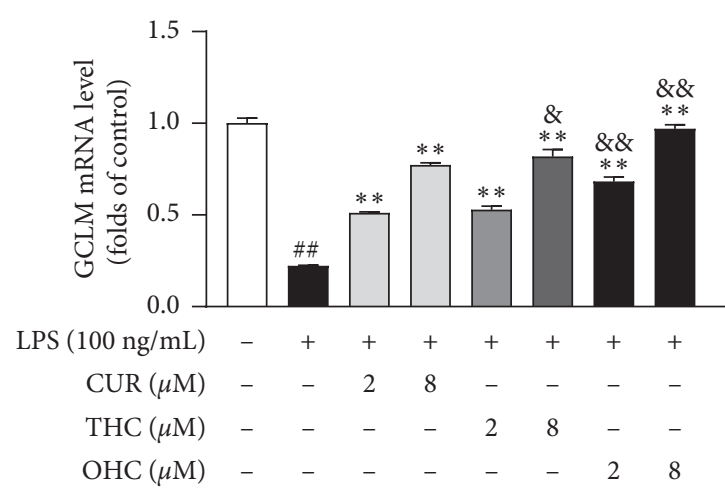

(d)

FIgURE 4: Effects of CUR, THC, and OHC on HO-1 (a), NQO-1 (b), GCLC (c), and GCLM (d) mRNA expression in LPS-stimulated RAW264.7 cells. Data are shown as mean $\pm \mathrm{SD}(n=3)$; ${ }^{\#} P<0.01$ vs. control group, ${ }^{* *} P<0.01$ vs. LPS group, ${ }^{\&} P<0.05$, and ${ }^{\& \&} P<0.01$ vs. CUR of the same dose.

3.5. Evaluation of NF- $\kappa B$ and MAPK Signaling Pathways in RAW264.7 Cells. As shown in Figures 6(a)-6(d), the protein expression levels of $\mathrm{p}-\mathrm{P} 38 / \mathrm{P} 38 \quad(P<0.01), \mathrm{p}$-ERK/ERK $(P<0.01)$, and NF- $\kappa \mathrm{B}(P<0.01)$ were dramatically increased after LPS stimulation. Nevertheless, treatment with CUR, THC, and OHC at various concentrations blocked P38 and ERK phosphorylation to a certain extent, and dose dependently arrested (all $P<0.01$ ) the activation of NF- $\kappa$ B. Impressively, THC and OHC exhibited more obvious effect than CUR in suppressing the protein expression of p-P38MAPK, p-ERK/ERK, and NF- $\kappa$ B (all $P<0.01$ ).

\section{Discussion}

Macrophages are important immune regulatory cells widely present in almost all body tissues and organs, which also participate in complicated inflammatory immune response [35]. LPS serving as an important component is present in the cell wall of Gram-negative bacteria, which can be widely used to stimulate macrophages to induce inflammation through binding to receptors on the membrane of macrophages [36]. LPS promotes macrophages to release numerous proinflammatory mediators and cytokines [37]. In addition, oxidative stress is also an important factor of boosting inflammation. Therefore, the current study was initiated to comparatively explore the anti-inflammatory and antioxidant activities of CUR and its two important hydrogenated metabolites of THC and OHC in LPS-stimulated RAW264.7 cells.

NO is a marker of proinflammatory mediator, and its production and formation can be mediated by iNOS catalyzing L-arginine [38]. iNOS, an important inflammatory factor, is activated in the inflammatory response. And excessive levels of iNOS-mediated NO production will exasperate inflammatory response [39]. Therefore, it is important to inhibit the excessive secretion of NO for controlling the inflammatory response. As our results indicated, pretreatment with CUR, THC, and OHC significantly inhibited NO production, which may be through downregulating iNOS mRNA expression. Importantly, it was noteworthy that THC and $\mathrm{OHC}$ exhibited a stronger inhibitory effect on iNOS-induced $\mathrm{NO}$ production than CUR, which was consistent with the finding of Zhao et al. [10].

Chemokines serve as important heparin-binding proteins that are released by macrophages, which are found to exert an important influence on various inflammatory diseases [40]. MCP-1 is one of the most representative chemokines which belongs to the CC family. It could implicate the inflammatory and immune reaction processes by mediating the infiltration and migration of monocytes/ macrophages [41, 42]. According to our results, OHC and THC also possessed appreciable effect to CUR in decreasing the accumulation and mRNA expression of MCP-1.

It is well-known that macrophages exposed to LPS can induce the activation of NF- $\kappa \mathrm{B}$ and MAPKs via TLR4mediated responses [43]. A bulk of evidence has proved that $\mathrm{NF}-\kappa \mathrm{B} / \mathrm{MAPKs}$ signaling are highly related to the inflammation-associated pathogenesis through regulating their downstream proinflammatory mediators [44]. To further examine the mechanism of CUR, THC, and OHC in LPSinduced inflammation, we investigated whether they would affect NF- $\kappa$ B and MAPKs (p38MAPK and ERK) signal pathways. As earlier reported, NF- $\kappa \mathrm{B}$ combines with its inhibitor kappa $\mathrm{B}(\mathrm{I} \kappa \mathrm{B} \alpha)$ in the cytoplasm under inactive conditions. After activated by LPS, I $\kappa \mathrm{B} \alpha$ is then phosphorylated and degraded, and the I $\kappa$ B kinases (IKKs) control this process [45]. Subsequently, the released NF- $\kappa$ B is then transferred from the cytosol to the nucleus, leading to the transcription and expression of numerous proinflammatory genes, as well as chemokines [46]. Therefore, inhibitor acting on NF- $\kappa \mathrm{B}$ activation is a potential therapeutic target in inflammatory response. In this work, CUR, THC, and OHC pretreatment dramatically inhibited NF- $\kappa \mathrm{B}$ activation.

In addition, massive reports have found that MAPKs consisted of four main families: p38MAPK, extracellular signal-regulated kinase (ERK), Jun N-terminal kinase (JNK), and ERK5 [47]. They are crucial for various inflammatory physiological processing, including regulating downstream gene expression [48]. Our results showed that the protein 


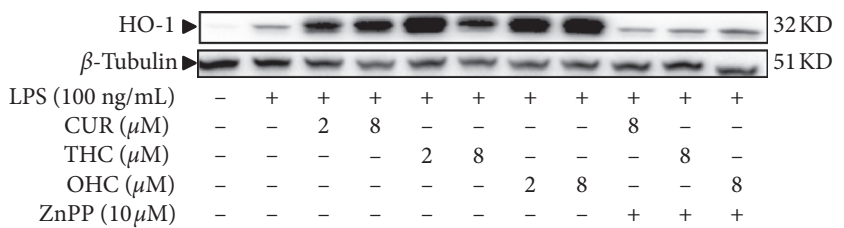

(a)

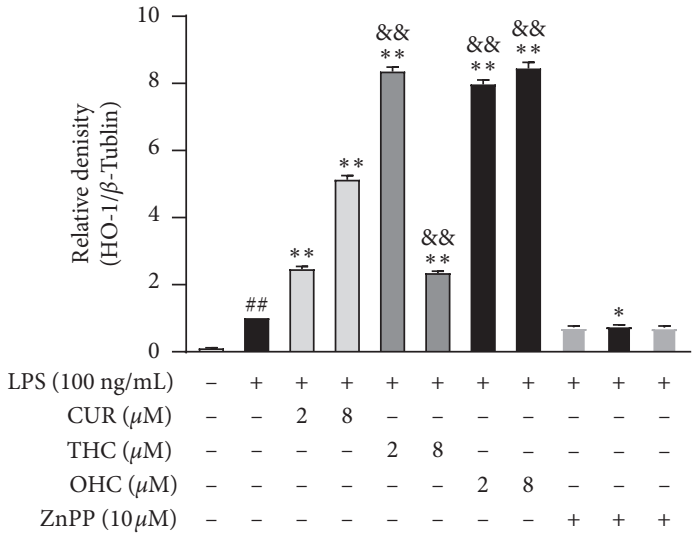

(b)

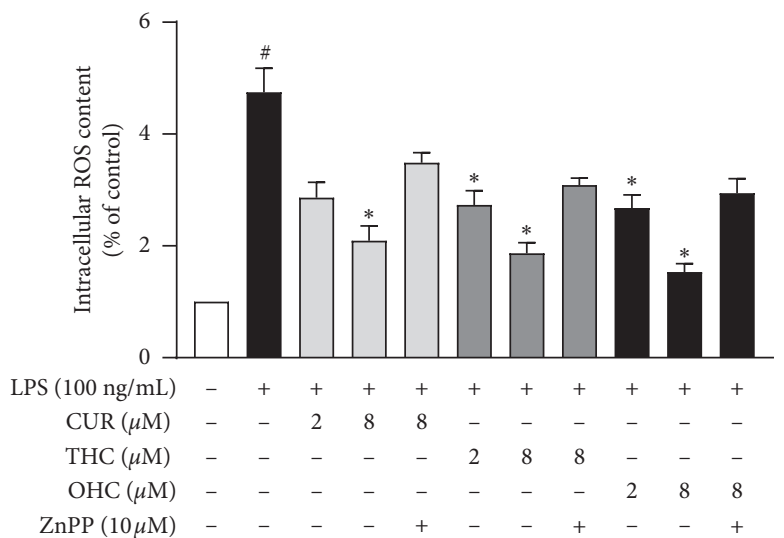

(c)

Figure 5: Effects of ZnPP on the generation of HO-1 and ROS by CUR, THC, and OHC in LPS-stimulated RAW264.7 cells. Data are shown as mean \pm SD $(n=3)$; ${ }^{\# \#} P<0.01$ vs. control group, ${ }^{*} P<0.05,{ }^{* *} P<0.01$ vs. LPS group, and ${ }^{\& \&} P<0.01$ vs. CUR of the same dose.

expression levels of $\mathrm{p}$-P38/P38 and p-ERK/ERK were obviously enhanced in the presence of LPS. However, pretreatment with CUR, THC, and OHC dose dependently suppressed the phosphorylation of both p-ERK and p-P38. Taken together, these results strongly suggested that CUR, THC, and OHC significantly suppressed NO and MCP-1 synthesis, and iNOS and MCP-1 mRNA expression, which might be closely related to the inhibition of NF- $\kappa \mathrm{B} / \mathrm{MAPKs}$ activation. Importantly, the relative potency of $\mathrm{OHC}$ and $\mathrm{THC}$ for repressing NF- $\kappa \mathrm{B} / \mathrm{MAPKs}$ activation was superior to that of CUR.

It has been reported that high levels of reactive oxygen species (ROS) will cause redox imbalance in vivo, which leads to oxidative stress [49]. Nrf2 is an important nuclear transcription factor belonging to basic-leucine zipper transcription factor family [50]. Furthermore, as an important endogenous antioxidant pathway, Nrf2 has a major role in improving cellular antioxidant response through regulating the antioxidant and cytoprotective gene expression [51, 52]. Under basal circumstances, the Kelch-like ECH-associated protein 1 (Keap1), serving as an important repressor, is associated with Nrf2. While under stressful conditions, the complex degrades and Nrf2 evades repression by Keap1, allowing its translocation from cytoplasm to nucleus [53]. Then, the leased Nrf2 combines with antioxidant response element (ARE), which promotes the expression of Nrf2-regulated genes [16, 54]. Luo et al. indicated that CUR, THC, and OHC could activate Nrf2 signaling pathway and increase its downstream ARE-driven gene expression [16]. We showed that CUR, THC, and OHC were able to markedly upregulate the cytoprotective gene expression (HO-1, NQO-1, GCLC, and GCLM) in LPSstimulated RAW264.7 macrophages, which was consistent with previous studies $[16,55]$. Interestingly, $\mathrm{OHC}$ and $\mathrm{THC}$ also possessed superior effect in protecting these gene expressions to CUR.

HO-1 is a rate-limiting enzyme, which can be used to produce carbon monoxide (CO) and biliverdin through degrading the pro-oxidant heme [56]. It has been shown that HO-1 has a key influence in mediating the antioxidant tissue damage $[57,58]$. In order to verify whether the alleviative effect of CUR, THC, and OHC on ROS generation was mediated by HO-1, ZnPP (a HO-1 inhibitor) was applied. We found that ROS production was obviously potentiated in the presence of CUR, THC, and OHC, respectively. However, the protective effect was reserved by $\mathrm{ZnPP}$ to a certain degree. This meant that upregulating HO-1 mRNA expression contributed to the ROS reduction in LPS-induced RAW 264.7 cells.

Overall, our experimental findings indicated that THC and $\mathrm{OHC}$ were found to exhibit superior effect to CUR in alleviating NF- $\kappa \mathrm{B} / \mathrm{MAPKs}-$ mediated inflammatory response 


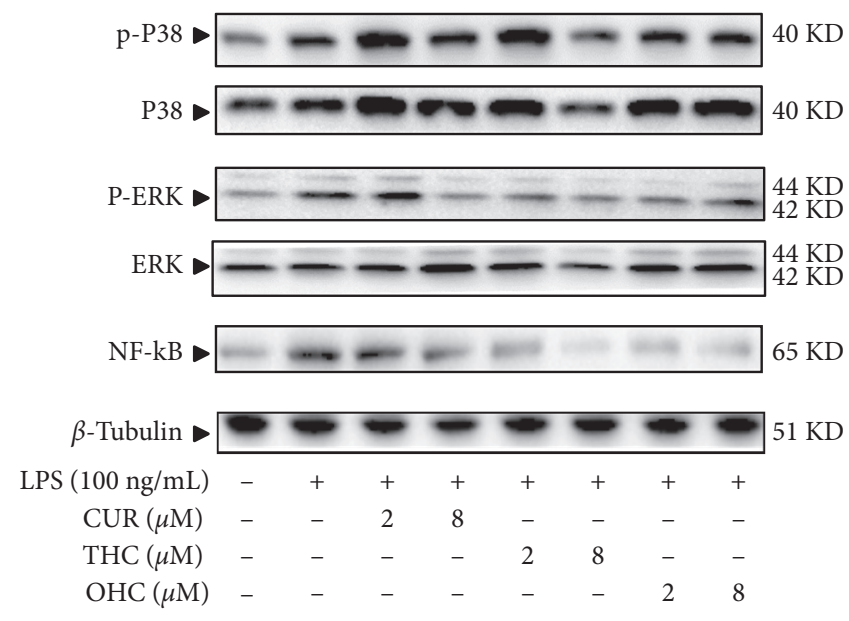

(a)

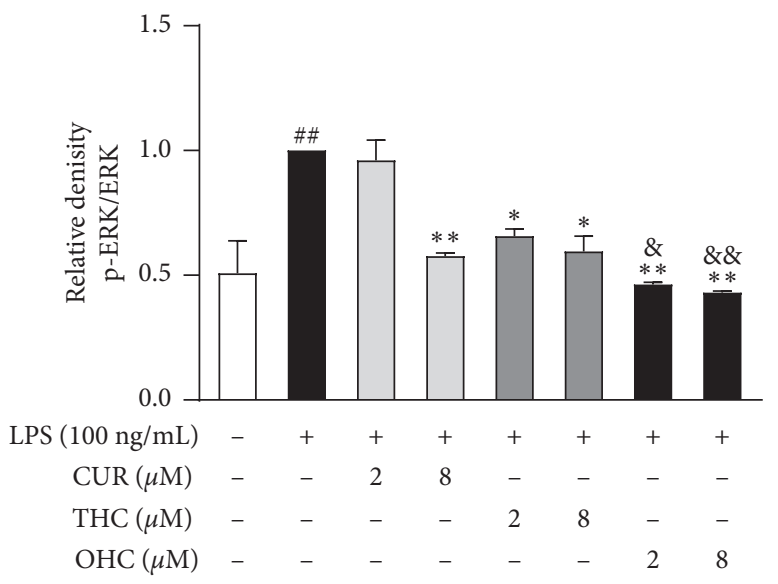

(c)

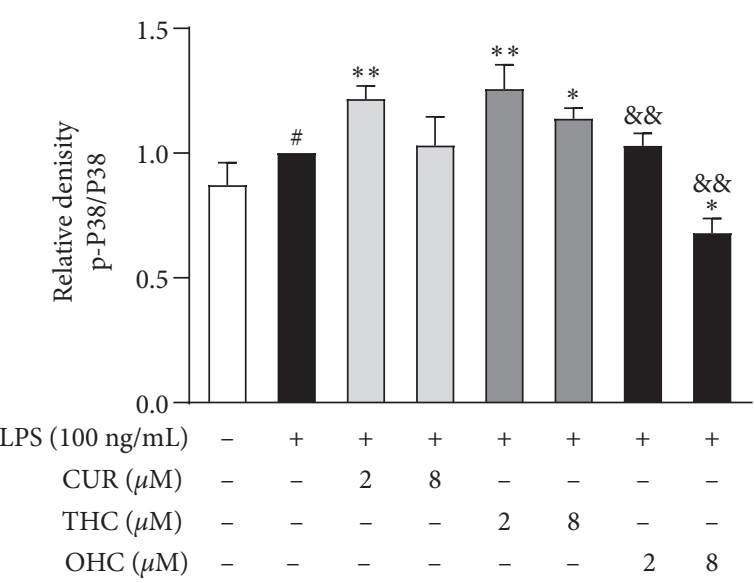

(b)

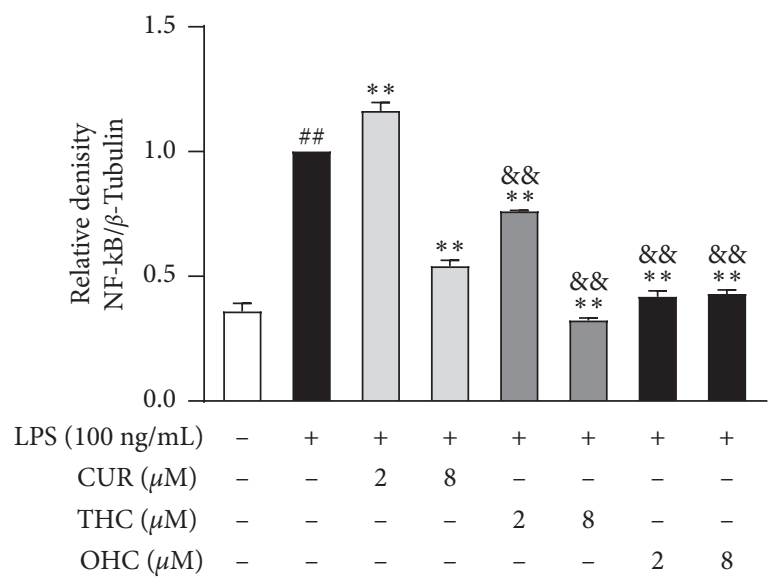

(d)

Figure 6: Effects of CUR, THC, and OHC on protein expression levels of p-P38/P38 (b), p-ERK/ERK (c), and NF- $\kappa$ B (d) in LPS-stimulated RAW264.7 cells. (a) The representative expression bands. Data are shown as mean $\pm \operatorname{SD}(n=3) ;{ }^{\# \#} P<0.01$ vs. control group, ${ }^{* *} P<0.01$ vs. LPS group, ${ }^{\&} P<0.05$, and ${ }^{\& \&} P<0.01$ vs. CUR of the same dose.

and upregulating Nrf2-regulated antioxidant genes. These may be correlated with their structural differences. CUR has $\alpha, \beta$ dienes, while THC and OHC do not. Hydrogenation of the heptadiene moiety and $\beta$-diketone of CUR to THC and OHC can substantially potentiate the antioxidant and antiinflammatory activities.

\section{Conclusions}

Taken together, the current experimental study clearly exhibited anti-inflammatory and antioxidant activities of CUR, THC, and OHC in a model of LPS-induced RAW264.7 cells. We demonstrated that the anti-inflammatory mechanism was associated with modulation of NF- $\kappa \mathrm{B}$ and MAPKs signaling pathways. And the antioxidant effects may be related to activating Nrf2-regulated downstream antioxidant genes. Furthermore, our findings also offer some significant and valuable experimental evidence that $\mathrm{THC}$ and $\mathrm{OHC}$ exerted more pronounced anti-inflammatory and antioxidant activities to CUR in vitro, implying that THC and $\mathrm{OHC}$ might be the important bioactive anti-inflammatory and antioxidant forms of CUR in vivo. This work is envisaged to provide further insight into the potential of $\mathrm{THC}$ and $\mathrm{OHC}$ as promising therapeutic agents against inflammatory diseases. In the following endeavor, further in-depth investigation should be merited to provide more enlightening dimensions.

\section{Data Availability}

The data used to support the findings of this study are available from the corresponding author upon request.

\section{Conflicts of Interest}

The authors declare that they have no conflicts of interest.

\section{Authors' Contributions}

Qing-Feng Xie and Juan-Juan Cheng contributed equally to this work. Q.-F.X. and J.-J.C. conceptualized the study; J.F.C. and G.-S.L. contributed to methodology; J.-J.C. and Y.C.F. were responsible for formal analysis; Q.-F.X. and J.-J.C. contributed to writing-original draft preparation; Y.X. 
contributed to writing-original draft preparation; Q.-F.X. and Y.X. were responsible for funding acquisition.

\section{Acknowledgments}

This research was supported by the Hospital Project of Guangdong Provincial Hospital of Chinese Medicine (no. E40404).

\section{References}

[1] N. Chainani-Wu, "Safety and anti-inflammatory activity of curcumin: a component of tumeric (Curcuma longa)," Journal of Alternative and Complementary Medicine, vol. 1, no. 9, pp. 161-168, 2003.

[2] J. H. Lim and T. K. Kwon, "Curcumin inhibits phorbol myristate acetate (PMA)-induced MCP-1 expression by inhibiting ERK and NF- $\kappa \mathrm{B}$ transcriptional activity," Food and Chemical Toxicology, vol. 48, no. 1, pp. 47-52, 2009.

[3] S. G. Han, J. Xu, X. J. Guo, and M. Huang, "Curcumin ameliorates severe influenza pneumonia via attenuating lung injury and regulating macrophage cytokines production," Clinical and Experimental Pharmacology \& Physiology, vol. 45, no. 1, pp. 84-93, 2018.

[4] A. Milad, Y. Habib, and S. Amirhossein, "Therapeutic effects of curcumin against bladder cancer: a review of possible molecular pathways," Anti-Cancer Agents in Medicinal Chemistry, vol. 20, no. 6, pp. 667-677, 2020.

[5] A. Shehzad, G. Rehman, and Y. S. Lee, "Curcumin in inflammatory diseases," Biofactors, vol. 39, no. 1, pp. 69-77, 2013.

[6] J. Yu, X. M. Zhou, X. S. He, M. Dai, and Q. Zhang, "Curcumin induces apoptosis involving bax/bcl-2 in human hepatoma SMMC-7721 cells," Asian Pacific Journal of Cancer Prevention: APJCP, vol. 12, no. 8, pp. 1925-1929, 2011.

[7] A. Preetha, P. Anand, A. B. Kunnumakkara, R. A. Newman, and B. B. Aggarwal, "Bioavailability of curcumin: problems and promises," Molecular Pharmaceutics, vol. 4, no. 6, pp. 807-818, 2007.

[8] W. Liu, Z. Zhang, G. Lin et al., "Tetrahydrocurcumin is more effective than curcumin in inducing the apoptosis of $\mathrm{H} 22$ cells via regulation of a mitochondrial apoptosis pathway in ascites tumor-bearing mice," Food \& Function, vol. 8, no. 9, pp. 3120-3129, 2017.

[9] K. Okada, C. Wangpoengtrakul, T. Tanaka et al., "Curcumin and especially tetrahydrocurcumin ameliorate oxidative StressInduced renal injury in mice," The Journal of Nutrition, vol. 131, no. 8, pp. 2090-2095, 2001.

[10] F. Zhao, Y. D. Gong, Y. Hu et al., "Curcumin and its major metabolites inhibit the inflammatory responseinduced by lipopolysaccharide: translocation of nuclear factor- $\kappa \mathrm{B}$ as potential target," Molecular Medicine Reports, vol. 11, no. 4, pp. 3087-3093, 2015.

[11] Z. B. Zhang, D. D. Luo, J. H. Xie et al., "Curcumin's metabolites, tetrahydrocurcumin and octahydrocurcumin, possess superior anti-inflammatory effects in vivo through suppression of TAK1-NF-kappaB pathway," Frontiers in Pharmacology, vol. 9, p. 1181, 2018.

[12] P. Murugan and L. Pari, "Influence of tetrahydrocurcumin on erythrocyte membrane bound enzymes and antioxidant status in experimental type 2 diabetic rats," Journal of Ethnopharmacology, vol. 113, no. 3, pp. 479-486, 2007.

[13] P. Somparn, C. Phisalaphong, S. Nakornchai, S. Unchern, and N. P. Morales, "Comparative antioxidant activities of curcumin and its demethoxy and hydrogenated derivatives," Biological and Pharmaceutical Bulletin, vol. 30, no. 1, pp. 74-78, 2007.

[14] S. Nakmareong, U. Kukongviriyapan, P. Pakdeechote et al., "Antioxidant and vascular protective effects of curcumin and tetrahydrocurcumin in rats with L-NAME-induced hypertension," Naunyn-Schmiedeberg's Archives of Pharmacology, vol. 383, no. 5, pp. 519-529, 2011.

[15] T. Osawa and Y. Kato, "Protective role of antioxidative food factors in oxidative stress caused by hyperglycemia," Annals of the New York Academy of Sciences, vol. 1043, no. 1, pp. 440-451, 2005.

[16] D. D. Luo, J. F. Chen, J. J. Liu et al., "Tetrahydrocurcumin and octahydrocurcumin, the primary and final hydrogenated metabolites of curcumin, possess superior hepatic-protective effect against acetaminophen-induced liver injury: role of CYP2E1 and Keap1-Nrf2 pathway," Food and Chemical Toxicology, vol. 123, pp. 349-362, 2019.

[17] D. L. Ambriz-Pérez, N. Leyva-López, J. P. Heredia, and E. P. Gutierrez-Grijalva, "Phenolic compounds: natural alternative in inflammation treatment. A review," Cogent Food \& Agriculture, vol. 2, no. 1, Article ID 1131412, 2016.

[18] J. Wong, B. Magun, and L. Wood, "Lung inflammation caused by inhaled toxicants: a review," International Journal of Chronic Obstructive Pulmonary Disease, vol. 11, pp. 13911401, 2016.

[19] S. J. Hwang, Y.-W. Kim, Y. Park, H. J. Lee, and K. W. Kim, "Anti-inflammatory effects of chlorogenic acid in lipopolysaccharide-stimulated RAW 264.7 cells," Inflammation Research, vol. 63, no. 1, pp. 81-90, 2014.

[20] I. T. Lee and C. M. Yang, "Inflammatory signalings involved in airway and pulmonary diseases," Mediators of Inflammation, vol. 2013, Article ID 791231, 12 pages, 2013.

[21] C. B. Ahn, Y. S. Cho, and J. Y. Je, "Purification and antiinflammatory action of tripeptide from salmon pectoral fin byproduct protein hydrolysate," Food Chemistry, vol. 168, pp. 151-156, 2015.

[22] Q. Xie, W. W. Shen, J. Zhong, C. Huang, L. Zhang, and J. Li, "Lipopolysaccharide/adenosine triphosphate induces IL1beta and IL-18 secretion through the NLRP3 inflammasome in RAW264.7 murine macrophage cells," International Journal of Molecular Medicine, vol. 34, no. 1, pp. 341-349, 2014.

[23] I. K. Madera-Salcedo, S. L. Cruz, and C. Gonzalez-Espinosa, "Morphine prevents lipopolysaccharide-induced TNF secretion in mast cells blocking I $\kappa$ B kinase activation and SNAP-23 phosphorylation: correlation with the formation of a $\beta$-arrestin/TRAF6 complex," The Journal of Immunology, vol. 191, no. 6, pp. 3400-3409, 2013.

[24] S. Uwe, "Anti-inflammatory interventions of NF- $\kappa$ B signaling: potential applications and risks," Biochemical Pharmacology, vol. 75, no. 8, pp. 1567-1579, 2008.

[25] C. Y. Zhang, S. Q. Lin, F. Y. Liu et al., “The anti-inflammatory effect of ent-kaur-15-en-17-al-18-oic acid on lipopolysaccharide-stimulated RAW264.7 cells associated with NF- $\kappa \mathrm{B}$ and P38/MAPK pathways," Journal of Asian Natural Products Research, vol. 22, pp. 1-14, 2020.

[26] Y. Shi, Y. Sun, X. P. Sun et al., "Up-regulation of HO-1 by Nrf2 activation protects against palmitic acid-induced ROS increase in human neuroblastoma BE (2)-M17 cells," Nutrition Research, vol. 52, pp. 80-86, 2018.

[27] R. Gill, A. Tsung, and T. Billiar, "Linking oxidative stress to inflammation: toll-like receptors," Free Radical Biology and Medicine, vol. 48, no. 9, pp. 1121-1132, 2010. 
[28] N. Truyen, N. Paul, and C. B. Pickett, "The Nrf2-antioxidant response element signaling pathway and its activation by oxidative stress," The Journal of Biological Chemistry, vol. 284, no. 20, pp. 13291-13295, 2009.

[29] J. W. Kaspar, S. K. Niture, and A. K. Jaiswal, "Nrf2:INrf2 (Keap1) signaling in oxidative stress," Free Radical Biology and Medicine, vol. 47, no. 9, pp. 1304-1309, 2009.

[30] N. S. Khurana and S. C. Sikka, "Targeting crosstalk between Nrf-2, NF-kappaB and androgen receptor signaling in prostate cancer," Cancers, vol. 10, no. 10, 352 pages, 2018.

[31] C. Li, T. Chen, H. Zhou et al., "Schisantherin A attenuates neuroinflammation in activated microglia: role of Nrf2 activation through ERK phosphorylation," Cellular Physiology and Biochemistry, vol. 47, no. 5, pp. 1769-1784, 2018.

[32] Z. B. Zhang, D. D. Luo, J. H. Xie et al., "Octahydrocurcumin, a final hydrogenated metabolite of curcumin, possesses superior anti-tumor activity through induction of cellular apoptosis," Food and Function, vol. 9, no. 4, pp. 2005-2014, 2019.

[33] Y.-F. Xian, Y.-C. Li, S.-P. Ip, Z.-X. Lin, X.-P. Lai, and Z.-R. Su, "Anti-inflammatory effect of patchouli alcohol isolated from pogostemonis herba in LPS-stimulated RAW264.7 macrophages," Experimental and Therapeutic Medicine, vol. 2, no. 3, pp. 545-550, 2011.

[34] C. Riebeling, J. P. Piret, B. Trouiller et al., "A guide to nanosafety testing: considerations on cytotoxicity testing in different cell models," NanoImpact, vol. 10, pp. 1-10, 2018.

[35] P. J. Murray and T. A. Wynn, "Protective and pathogenic functions of macrophage subsets," Nature Reviews Immunology, vol. 11, no. 11, pp. 723-737, 2011.

[36] T. Varadharajan, S. K. Selvi, V. V. Padma, and C. F. Weng, "16-hydroxy-cleroda-3,13-dien-16,15-olide induced glioma cell autophagy via ROS generation and activation of p38 MAPK and ERK-1/2," Environmental Toxicology and Pharmacology, vol. 45, pp. 202-211, 2016.

[37] B. O. Cho, D. N. Che, J. S. Kim et al., "In vitro anti-inflammatory and anti-oxidative stress activities of kushenol C isolated from the roots of Sophora flavescens," Molecules, vol. 25, no. 8, 1768 pages, 2020.

[38] H. H. Schmidt, R. Seifert, and E. Böhme, "Formation and release of nitric oxide from human neutrophils and HL-60 cells induced by a chemotactic peptide, platelet activating factor and leukotriene B4," FEBS Letters, vol. 244, no. 2, pp. 357-360, 1989.

[39] G. Y. Yang, S. Taboada, and J. Liao, "Induced nitric oxide synthase as a major player in the oncogenic transformation of inflamed tissue," Methods in Molecular Biology, vol. 512, pp. 119-156, 2009.

[40] G. Lazennec and A. Richmond, "Chemokines and chemokine receptors: new insights into cancer-related inflammation," Trends in Molecular Medicine, vol. 16, no. 3, pp. 133-144, 2010.

[41] S. L. Deshmane, S. Kremlev, S. Amini, and B. Sawaya, "Monocyte chemoattractant protein-1 (MCP-1): an overview," Journal of Interferon \& Cytokine Research, vol. 29, no. 6, pp. 313-326, 2009.

[42] J. Melchjorsen, L. N. Sørensen, and S. R. Paludan, "Expression and function of chemokines during viral infections: from molecular mechanisms to in vivo function," Journal of Leukocyte Biology, vol. 74, no. 3, pp. 331-343, 2003.

[43] D. H. Kwon, H. J. Cha, E. O. Choi et al., "Schisandrin A suppresses lipopolysaccharide-induced inflammation and oxidative stress in RAW 264.7 macrophages by suppressing the NFkappaB, MAPKs and PI3K/Akt pathways and activating Nrf2/ HO-1 signaling," International Journal of Molecular Medicine, vol. 41, no. 1, pp. 264-274, 2018.

[44] K. C. Lee, H. H. Chang, Y. H. Chung, and T. Y. Lee, "Andrographolide acts as an anti-inflammatory agent in LPS- stimulated RAW264.7 macrophages by inhibiting STAT3-mediated suppression of the NF- $\kappa \mathrm{B}$ pathway," Journal of Ethnopharmacology, vol. 135, no. 3, pp. 678-684, 2018.

[45] L. Y. Cui, H. Wang, J. Q. Lin et al., "Progesterone inhibits inflammatory response in E. coli-or LPS-Stimulated bovine endometrial epithelial cells by NF- $\kappa \mathrm{B}$ and MAPK pathways," Developmental and Comparative Immunology, vol. 105, Article ID 103568, 2020.

[46] W. F. Li, X. M. Wang, H. L. Zhang et al., "Anti-ulcerogenic effect of cavidine against ethanol-induced acute gastric ulcer in mice and possible underlying mechanism," International Immunopharmacology, vol. 38, pp. 450-459, 2016.

[47] S.-H. Yang, A. D. Sharrocks, and A. J. Whitmarsh, "MAP kinase signalling cascades and transcriptional regulation," Gene, vol. 513, no. 1, pp. 1-13, 2013.

[48] G. L. Johnson and R. Lapadat, "Mitogen-activated protein kinase pathways mediated by ERK, JNK, and p38 protein kinases," Science, vol. 5600298 pages, 2002.

[49] Y. Shi, Y. Sun, X. Sun et al., "Up-regulation of HO-1 by Nrf2 activation protects against palmitic acid-induced ROS increase in human neuroblastoma BE (2)-M17 cells," Nutrition Research, vol. 52, pp. 80-86, 2018.

[50] H. G. Chen, K. L. Xie, H. Z. Han et al., "Molecular hydrogen protects mice against polymicrobial sepsis by ameliorating endothelial dysfunction via an $\mathrm{Nrf} 2 / \mathrm{HO}-1$ signaling pathway," International Immunopharmacology, vol. 28, no. 1, pp. 643-654, 2015.

[51] Y.-J. Surh, J. Kundu, and H.-K. Na, "Nrf2 as a master redox switch in turning on the cellular signaling involved in the induction of cytoprotective genes by some chemopreventive phytochemicals," Planta Medica, vol. 74, no. 13, pp. 1526-1539, 2008.

[52] A. Loboda, M. Damulewicz, E. Pyza, A. Jozkowicz, and J. Dulak, "Role of Nrf2/HO-1 system in development, oxidative stress response and diseases: an evolutionarily conserved mechanism," Cellular and Molecular Life Sciences, vol. 73, no. 17, pp. 32213247, 2016.

[53] M. C. Jaramillo and D. D. Zhang, "The emerging role of the Nrf2Keap1 signaling pathway in cancer," Genes \& Development, vol. 27, no. 20, pp. 2179-2191, 2013.

[54] C. K. Kim, D. H. Cho, K. S. Lee et al., "Ginseng berry extract prevents atherogenesis via anti-inflammatory action by upregulating phase II gene expression," Evidence-Based Complementary and Alternative Medicine, vol. 2012, Article ID 490301, 14 pages, 2012.

[55] Y. Zhong, T. Liu, W. Lai, Y. Tan, D. Tian, and Z. Guo, "Heme oxygenase-1-mediated reactive oxygen species reduction is involved in the inhibitory effect of curcumin on lipopolysaccharide-induced monocyte chemoattractant protein-1 production in RAW264.7 macrophages," Molecular Medicine Reports, vol. 7, no. 1, pp. 242-246, 2013.

[56] S. W. Ryter, J. Alam, and A. M. K. Choi, "Heme oxygenase-1/ carbon monoxide: from basic science to therapeutic applications," Physiological Reviews, vol. 86, no. 2, pp. 583-650, 2006.

[57] H.-O. Pae, G.-S. Jeong, H.-S. Kim et al., "Costunolide inhibits production of tumor necrosis factor- $\alpha$ and interleukin- 6 by inducing heme oxygenase-1 in RAW264.7 macrophages," Inflammation Research, vol. 56, no. 12, pp. 520-526, 2007.

[58] K. M. Kim, H.-O. Pae, M. Zhung et al., "Involvement of antiinflammatory heme oxygenase- 1 in the inhibitory effect of curcumin on the expression of pro-inflammatory inducible nitric oxide synthase in RAW264.7 macrophages," Biomedicine \& Pharmacotherapy, vol. 62, no. 9, pp. 630-636, 2008. 